\title{
Avaliação sazonal do estado nutricional do algodoeiro herbáceo colorido BRS Rubi
}

\author{
Franciezer V. de Limaํ, José R. Pereira², Érica S. A. B. de Almeida ${ }^{3}$, Vandeilson L. Araújo, \\ Whéllyson P. Araújo ${ }^{3}$, Severino P. de Souza Junior
}

\begin{abstract}
1 Universidade Federal Rural do Semiárido, Departamento de Ciências Ambientais e Tecnológicas, Programa de Pós Graduação em Manejo de Solo e Agua, Av. Francisco Mota, 572, Costa e Silva, CEP 59.625-900, Mossoró- RN, Brasil. Caixa Postal 137. E-mail: franciezer@hotmail.com

2 Empresa Brasileira de Pesquisa Agropecuária, Embrapa Algodão, Campina Grande, PB, Rua Oswaldo Cruz, 1143, Centenário, Caixa Postal 174. CEP: 58.428-095, E-mail: jose.r.pereira@embrapa.br

${ }^{3}$ Universidade Federal de Campina Grande, Programa de Pós-Graduação em Engenharia Agrícola, Rua Aprígio Veloso, 882, Bairro Universitário, Campina Grande, PB, CEP 58.429-900, E-mail: ericasamara@gmail.com; wpacordao@hotmail.com

${ }^{4}$ Universidade Federal da Paraíba, Centro de Ciências Agrárias, Programa de Pós-Graduação em Agronomia, Campus II Areia, PB, BR 079, Km 12, CEP: 53.397-000, E-mail: vandeilsonlemos@hotmail.com

${ }^{5}$ Universidade Federal da Paraíba, Centro de Ciências Agrárias, Departamento de Fitotecnia e Ciências Ambientais, Campus II Areia, PB, BR 079, Km 12, CEP: 53.397-000, E-mail: severo-ita@bol.com.br
\end{abstract}

\section{RESUMO}

O conhecimento do estado nutricional das plantas é importante para que se possa avaliar o potencial de produção e buscar um equilíbrio entre os nutrientes. Estudos utilizando a diagnose foliar têm sido eficientes pois a planta é o próprio extrator de nutrientes do solo. Portanto, o objetivo deste trabalho foi verificar o estado nutricional e a redistribuição de nutrientes em plantas do algodoeiro herbáceo colorido cultivar BRS Rubi através da ferramenta da diagnose foliar. $O$ experimento foi conduzido na comunidade São Pedro, município de Itaporanga, PB, situado na mesorregião do Sertão Paraibano, no período de outubro de 2009 a janeiro de 2010. O delineamento experimental utilizado foi o de blocos ao acaso com quatro repetições, no esquema de parcelas subdivididas, cujas parcelas foram representadas por duas épocas de amostragem de folhas (aos 58 e 107 dias após o plantio - DAP) e as subparcelas por seis níveis de adubação orgânica na forma de doses crescentes de esterco bovino (0; 2,5; 5; 10; 20 e 40 t ha $^{-1}$ ). Apenas o N o K, determinados aos 58 e 107 dias após o plantio, apresentaram significância e maior redistribuição quando comparados aos demais macronutrientes. Para os micronutrientes analisados não foi observada diferença significativa entre os períodos avaliados.

Palavras-chave: avaliação nutricional, BRS Rubi, Gossypium hirsutum L. r. latifolium H., translocação de nutrientes

\section{Seasonal evaluation of colored upland cotton BRS Rubi nutritional status}

\begin{abstract}
The knowledge of the plants nutritional status is important to evaluate the production potential and to search for a balance between nutrients. Studies using foliar diagnosis have been very efficient, because the plant is the soil nutrient extractor itself. Therefore, the aim of this study was to determine the nutritional status and the redistribution of nutrients in plants of the colored upland cotton cultivar BRS Rubi by foliar analysis tool. The experiment was conducted in São Pedro community, Itaporanga County, Paraíba State, Brazil, located in the Sertão Paraibano mesoregion, from October 2009 to January 2010. The experimental design was a randomized block with four replications in a split plot scheme, where plots were represented by two periods of sampling leaves (at 58 and 107 days after planting - DAP) and the subplots by six levels of organic adubation in the form of increasing doses of bovine manure $\left(0 ; 2.5 ; 5 ; 10 ; 20\right.$ and 40 t ha $\left.^{-1}\right)$. Only the $\mathrm{N}$ and $\mathrm{K}$ determined at 58 and 107 days after planting, presented significant and greater redistribution compared to the other macronutrients analyzed. For the micronutrients analyzed, no significant difference between the evaluation periods was observed.
\end{abstract}

Key words: nutritional assessment, BRS Rubi, Gossypium hirsutum L. r. latifolium H., translocation of nutrients 


\section{Introdução}

O algodoeiro produz grande infinidade de usos, tendo destaque para sua fibra no caráter têxtil, além de oferecer numerosas possibilidades de aproveitamento para seus subprodutos, notadamente o óleo e a torta, provenientes das sementes (Silva, 2006). O conhecimento dos teores de nutrientes em vários estádios e nos órgãos do vegetal permite inferir sobre as exigências metabólicas e nutricionais desenvolvidas em cada compartimento, fornecendo base para o entendimento dessas variações e suas implicações nas respostas fisiológicas dos vegetais no campo (Brandão et al., 2012).

O conhecimento do estado nutricional das plantas é relevante para que se possa avaliar o potencial de produção e buscar um equilíbrio entre os nutrientes. Estudos utilizando a diagnose foliar têm sido bastante eficientes, visto que a planta é o próprio extrator de nutrientes do solo, possibilitando um diagnóstico nutricional direto e preciso (Lima et al., 2011).

De acordo com Morais (2010) a variação sazonal na concentração dos elementos minerais nas folhas parece estar fortemente relacionada aos mecanismos de translocação e redistribuição desses nutrientes. A interpretação correta dos resultados de análises foliares proporciona informações que favorecem o uso racional de insumos, evita desperdícios, melhora o equilíbrio nutricional das plantas e proporciona aumento da produtividade (Politi et al., 2013).

No caso da produção de algodão (Gossypium hirsutum L. r. latifolium H.), o estado nutricional das plantas assume ainda mais importância, com base nos efeitos dos nutrientes quanto ao aspecto fundamental para esta fibrosa: a qualidade das fibras. Cerca de $70 \%$ dos nutrientes são absorvidos após o aparecimento dos primeiros botões florais e cerca de $50 \%$ de todos os nutrientes são absorvidos no período que vai do florescimento à maturação (Silva, 1996); isto sugere que a formação do fruto do algodoeiro depende mais da absorção de nutrientes do solo que da sua redistribuição dentro das plantas (Silva, 2006).

A diagnose foliar é uma ferramenta de grande utilidade na avaliação do estado nutricional das plantas, de vez que as folhas são os órgãos que mais informações podem proporcionar com relação aos aspectos fisiológicos da planta (Sousa et al., 2012).

Ante o exposto, o objetivo deste trabalho foi verificar o estado nutricional e a redistribuição de nutrientes em plantas do algodoeiro herbáceo colorido cultivar BRS Rubi, através da ferramenta da diagnose foliar.

\section{Material e Métodos}

O experimento foi conduzido na comunidade São Pedro, município de Itaporanga, PB, situado na mesorregião do Sertão Paraibano, geograficamente localizado na latitude de $07^{\circ} 18^{\circ}$ $16^{`}$ Sul, na longitude de $38^{\circ} 09^{`} 01^{`}$ Oeste e na altitude 291 metros acima do nível do mar (Ramos et al., 2009), no período de 07 de outubro de 2009 a 17 de janeiro de 2010.

O preparo do solo constou de uma aração e duas gradagens tratorizadas. A irrigação foi realizada por aspersão convencional fixa, utilizando-se aspersor de 1/2" e se aplicando uma lâmina total de $520 \mathrm{~mm}$ durante o ciclo do algodoeiro. As capinas
Tabela 1. Características químicas da área experimental e do esterco bovino. Fazenda São Pedro, Itaporanga, PB, 2009/2010

\begin{tabular}{cccc}
\hline Características & Solo $^{(1)}$ & Características & Esterco $^{(2)}$ \\
\hline $\mathrm{pH}$ & 6,9 & Umidade $(\%)$ & 8,5 \\
Matéria Orgânica $\left(\mathrm{g} \mathrm{kg}^{-1}\right)$ & 17,4 & Matéria Orgânica $(\%)$ & 48,3 \\
$\mathrm{Ca}^{+2}\left(\mathrm{mmol}_{\mathrm{c}} \mathrm{dm}^{-3}\right)$ & 96,9 & Nitrogênio $(\%)$ & 2,0 \\
$\mathrm{Mg}^{+2}\left(\mathrm{mmol}_{\mathrm{c}} \mathrm{dm}^{-3}\right)$ & 22,1 & $\mathrm{P}_{2} \mathrm{O}_{5}(\%)$ & 1,2 \\
$\mathrm{Na}^{+}\left(\mathrm{mmol}_{\mathrm{c}} \mathrm{dm}^{-3}\right)$ & 0,8 & $\mathrm{~K}_{2} \mathrm{O}(\%)$ & 3,3 \\
$\mathrm{~K}^{+}\left(\mathrm{mmol}_{\mathrm{c}} \mathrm{dm}^{-3}\right)$ & 3,0 & Enxofre $(\%)$ & 0,2 \\
$\mathrm{Al}^{+3}\left(\mathrm{mmol}_{\mathrm{c}} \mathrm{dm}^{-3}\right)$ & 0,0 & $\mathrm{P}\left(\mathrm{mg} \mathrm{dm}^{-3}\right)$ & 479,2 \\
\hline
\end{tabular}

Boletim №. 157/2008 $8^{(1)}$ e №. 027/2008 ${ }^{(2)}$ do Laboratório de Solos e Nutrição de Plantas da Embrapa Algodão (Silva, 2009); M.O. = Matéria Orgânica

foram feitas manualmente. Não foi registrada a presença de bicudo e a infestação de cochonilha foi baixa, não atingindo o nível de controle.

O delineamento experimental utilizado foi o de blocos ao acaso, com quatro repetições, no esquema de parcelas subdivididas, em que as parcelas foram representadas por duas épocas de amostragem de folhas para a análise da nutrição mineral, aos 58 e 107 dias após o plantio (DAP) e as subparcelas por seis níveis de adubação orgânica na forma de doses crescentes de esterco bovino $\left(0 ; 2,5 ; 5 ; 10 ; 20 \mathrm{e} 40 \mathrm{t} \mathrm{ha}^{-1}\right)$ aplicado e incorporado em área total por ocasião da primeira capina, aos 20 DAP.

Nesse estudo foi utilizada a cultivar BRS Rubi de algodoeiro herbáceo colorido, cada parcela experimental era composta por uma área de $6,0 \mathrm{~m}$ de comprimento e $5,0 \mathrm{~m}$ de largura totalizando $30 \mathrm{~m}^{2}$, contendo cinco fileiras de algodão espaçadas entre si $1,0 \mathrm{~m}$ e dentro de cada fileira o espaçamento entre as plantas foi de $0,4 \mathrm{~m}$. A área útil da parcela foi de $10 \mathrm{~m}^{2}$, formada por duas linhas da área útil descontado $0,5 \mathrm{~m}$ de cada um dos lados para fins de bordadura. Aos 58 e 107 DAP foram coletadas todas as folhas de 5 plantas da área útil da parcela, colocadas em sacos de papel previamente identificados e secadas em estufa com circulação de ar, na temperatura $65^{\circ} \mathrm{C}$, por 48 horas.

Posteriormente, foram enviadas ao Laboratório do Instituto Agronômico de Pernambuco (IPA) para as análises de macro $(\mathrm{N}, \mathrm{P}, \mathrm{K}, \mathrm{Ca}, \mathrm{Mg})$ e micronutrientes ( $\mathrm{Fe}, \mathrm{Cu}$ e $\mathrm{Mn}$ ) adotandose as metodologias contidas em Silva (2009). De posse dos resultados, realizaram-se análise de variância, teste de $\mathrm{F}$ para parcelas (épocas de amostragem) e análise de regressão para as subparcelas (doses de esterco bovino). Utilizou-se o programa Assistat (Silva \& Azevedo, 2002).

\section{Resultados e Discussão}

Na análise de variância (Tabela 2), é possível observar diferença significativa entre as duas épocas de amostragem, apenas para as variáveis teores de $\mathrm{N}$ e $\mathrm{K}$ nas folhas do algodoeiro.

Os teores foliares significativamente maiores de $\mathrm{N}$ e $\mathrm{K}$ no algodoeiro herbáceo colorido cultivar BRS Rubi, foram observados durante a primeira amostragem, aos 58 DAP. Para os outros macronutrientes analisados $(\mathrm{P}, \mathrm{Ca}$ e $\mathrm{Mg}$ ) não houve diferença significativa entre as duas épocas de amostragem. Segundo Malavolta et al. (2004), os teores foliares de N, P, $\mathrm{K}, \mathrm{Ca}, \mathrm{Mg}$ e $\mathrm{S}$ variam em função do estádio fenológico da planta. 
Tabela 2. Análise de variância dos teores foliares de macronutrientes $\left(\mathrm{g} \mathrm{kg}^{-1}\right)$, em duas épocas de amostragem do algodoeiro herbáceo colorido BRS Rubi. Itaporanga-PB, 2009/2010

\begin{tabular}{|c|c|c|c|c|c|c|}
\hline FV & GL & $\mathbf{N}$ & $\mathbf{P}$ & $\mathbf{K}$ & $\mathrm{Ca}$ & Mg \\
\hline Blocos & 3 & 12890,97 & 498293,57 & 763103,07 & 1767457,63 & 3820889 \\
\hline Épocas (E) & 1 & $3980342,75^{\star *}$ & $13,02^{\text {ns }}$ & $528510950^{\star *}$ & $22102,08^{\text {ns }}$ & $19602,08^{\text {ns }}$ \\
\hline Resíduo a & 3 & 333840,97 & 454336,63 & 668694,46 & 1752702,08 & 5917,36 \\
\hline Parcelas & 7 & -- & -- & -- & -- & - \\
\hline Doses (D) & 5 & $341712,08 \mathrm{~ns}$ & 23931,77 ns & 570705,92 ns & $107433,08 \mathrm{~ns}$ & 1873,33 ns \\
\hline Int. (Ex D) & 5 & $400488,75^{\text {ns }}$ & 35209,27 ns & 600632,42 ns & $684267,08 \mathrm{~ns}$ & $2343,33 \mathrm{~ns}$ \\
\hline Resíduo b & 30 & 344329,30 & 156013,02 & 368088,40 & 449979,86 & 2957,50 \\
\hline Total & 47 & - & - & - & -- & - \\
\hline Média Geral & -- & 29,36 & 7,025 & 45,58 & 38,41 & 2,07 \\
\hline CV a $(\%)$ & - & 19,67 & 95,93 & 17,93 & 34,46 & 37,10 \\
\hline CV b $(\%)$ & -- & 19,98 & 56,21 & 13,30 & 17,46 & 26,23 \\
\hline
\end{tabular}

", ns: Significativos a 5\% e 1\% de probabilidade e não significativo, respectivamente; CV = Coeficiente de variação; Int = interação

A maior proporção dos nutrientes absorvidos durante o ciclo da planta se concentra durante a etapa de desenvolvimento vegetativo em que as plantas do algodoeiro estão formando sua massa verde para, posteriormente e durante o período de formação e enchimento das maçãs, translocar açúcares e nutrientes para os ramos reprodutivos (Rosolem et al., 2003).

De acordo com Severino et al. (2004) a vantagem do uso de adubo orgânico em relação à aplicação de fertilizantes químicos é a liberação gradual dos nutrientes à medida que são demandados para o crescimento da planta.

$\mathrm{Se}$ os nutrientes forem imediatamente disponibilizados no solo, como ocorre com os fertilizantes químicos, podem ser perdidos por volatilização $(\mathrm{N})$, fixação $(\mathrm{P})$ ou lixiviação $(\mathrm{N}$ e K). $\mathrm{O}$ processo de mineralização dos diferentes adubos orgânicos tem seu início a partir dos 20 ou 30 dias após sua incorporação ao solo (Severino et al., 2004). Este tempo vai depender principalmente da relação $\mathrm{C} / \mathrm{N}$ e do teor de $\mathrm{N}$ do adubo orgânico e das condições climáticas do meio, especialmente da temperatura e da umidade as quais vão estimular a atividade microbiana no solo (Souto et al., 2005).

A partir dos valores médios observados na Tabela 3 podese constatar que apenas os nutrientes $\mathrm{N}$ e $\mathrm{K}$ apresentaram significância em relação às épocas de amostragem, destacandose principalmente o $\mathrm{K}$, que teve valor médio aproximadamente seis vezes superior aos 58 dias quando comparado com a segunda época aos 107 DAP. Relatando, assim, a forte redistribuição que ocorreu com este nutriente, fato que pode ser atribuído à translocação necessária à formação dos botões florais e ao enchimento das maçãs, em que tal processo só ocorreu após a primeira época amostral cujos resultados são semelhantes aos encontrados por Motomiya et al. (2012) e Garrido et al. (2009).

De acordo com Marenco \& Lopes (2005) a dinâmica dos nutrientes em espécies vegetais varia em função da idade da planta, do estádio fenológico, condições edafoclimáticas e práticas de manejo adotadas e ao longo do ciclo fenológico a concentração de alguns elementos nas folhas aumenta enquanto a de outros decresce, ocorrendo translocação de nutrientes de órgãos senescentes para regiões de crescimento das plantas, como folhas novas e as estruturas reprodutivas, fato este observado por Lima et al. (2011) em estudo sobre a redistribuição de nutrientes em folhas de pinhão-manso entre estádios fenológicos.

Para Laviola et al. (2006) os elementos de alta mobilidade no floema, tais como $\mathrm{N}$ e $\mathrm{K}$, se apresentaram em maiores
Tabela 3. Médias dos teores foliares de macronutrientes $\left(\mathrm{g} \mathrm{kg}^{-1}\right)$, em duas épocas de amostragem, do algodoeiro herbáceo colorido BRS Rubi. Itaporanga-PB, 2009/2010

\begin{tabular}{cccccc}
\hline Épocas & $\mathbf{N}$ & $\mathbf{P}$ & $\mathbf{K}$ & $\mathbf{C a}$ & $\mathbf{M g}$ \\
\hline 58 DAP & $38,47 \mathrm{a}$ & $7,03 \mathrm{a}$ & $78,76 \mathrm{a}$ & $38,63 \mathrm{a}$ & $2,27 \mathrm{a}$ \\
107 DAP & $20,25 \mathrm{~b}$ & $7,02 \mathrm{a}$ & $12,40 \mathrm{~b}$ & $38,20 \mathrm{a}$ & $1,87 \mathrm{a}$ \\
\hline
\end{tabular}

Médias seguidas pela mesma letra nas colunas não diferem entre si pelo teste de $\mathrm{F}$ a $5 \%$ de probabilidade; $\mathrm{DAP}=$ dias após o plantio

quantidades (26,4 para 22,4 $\mathrm{g} \mathrm{kg}^{-1}$ de N e de 25,6 para 1,68 $\mathrm{g} \mathrm{kg}^{-1}$ de K) nas folhas, no início de seu desenvolvimento e exibiram um comportamento de redistribuição desses elementos das folhas para os órgãos reprodutivos e posteriormente frutos. Entretanto, nutrientes de baixa mobilidade, como $\mathrm{Ca}$ e $\mathrm{Mg}$, tendem a se concentrar em tecidos mais velhos visto que possuem baixa capacidade de redistribuição (Laviola et al., 2006).

Diante desses fatos constatou-se, neste estudo, que os teores médios de $\mathrm{K}$ foram altos na primeira amostragem visto que as plantas estavam em processo de crescimento e os mesmos foram reduzidos com o avanço das fases fenológicas, verificando-se este fato pelos valores médios encontrados nas épocas amostrais $\left(78,76\right.$ e 12,40 $\mathrm{g} \mathrm{kg}^{-1}$ aos 58 e 107 DAP respectivamente), Garrido et al. (2009) encontraram tendência semelhante $\left(58,0\right.$ e $\left.23,8 \mathrm{~g} \mathrm{~kg}^{-1}\right)$ para a cultura do algodoeiro adubado com diferentes fontes de matéria orgânica. Como já relatado, o K tem alta mobilidade no floema (Serra et al., 2010a; Marschner, 2002). Sendo conhecido na literatura que a maior parte do K é absorvida pelas plantas durante a fase de crescimento vegetativo, ou seja, na fase inicial de crescimento pois seu papel é fundamental na fotossíntese e na síntese de carboidratos (Lima et al., 2011).

Este nutriente possui papel importante na formação dos frutos atuando no transporte de fotoassimilados no floema (Laviola \& Dias, 2008), em que a deposição de biomassa no fruto é acompanhada, necessariamente, pelo acúmulo de K. Além disto, este é um nutriente requerido na ativação de diversas enzimas essenciais à síntese de compostos orgânicos (Marenco \& Lopes, 2005; Laviola \& Dias, 2008).

Através dos resultados deste estudo pode-se constatar que os teores de $\mathrm{K}$ foram mais altos no período inicial, no qual a atividade fisiológica é mais intensa (como fotossíntese) mas, à medida em que esses processos sofreram redução, o $\mathrm{K}$ foi redistribuído para outras partes da planta (órgãos reprodutivos).

Em relação ao $\mathrm{N}$ também se observa que houve uma concentração maior na primeira época amostral, quando 
comparada com a segunda, mais não tão evidente quando confrontado com o K. Sendo necessário destacar o N como nutriente essencial para a assimilação do $\mathrm{C}$ e a formação de novos órgãos na planta (Serra et al., 2010b; Motomiya et al., 2012).

Os teores foliares de $\mathrm{N}$ foram mais altos na primeira época de amostragem com valores médios de $38,47 \mathrm{~g} \mathrm{~kg}^{-1}$ e diminuíram significativamente entre a segunda época de amostragem $\left(20,25 \mathrm{~g} \mathrm{~kg}^{-1}\right)$ valores esses com tendência semelhante aos encontrados por Camacho et al. (2012) com médias de 43,0 para 30,1 $\mathrm{g} \mathrm{kg}^{-1}$. Dessalta-se que o nitrogênio tem alta mobilidade no floema (Marschner, 2002) e se espera que seu teor seja menor nos tecidos mais velhos ou em senescência, como era o caso da grande parte do material coletado para ser analisado na segunda época já que este nutriente pode ser redistribuído para outros órgãos, antes da queda da folha. No entanto, a redução no teor de N, entre folhas jovens e senescentes, embora estatisticamente significativa, é relativamente pequena e proporciona pouca redistribuição do nutriente, ou seja, a maior parte do $\mathrm{N}$ ao invés de estar sendo redistribuída na planta pode ter sido perdida pela queda da folha. Resultados similares aos observados neste estudo foram constatados por Morais (2010).

Os demais macronutrientes $(\mathrm{P}, \mathrm{Ca}, \mathrm{Mg})$ se mantiveram em equilíbrio nas duas épocas apontando, assim, que sua redistribuição foi menos evidente do que os nutrientes anteriormente discutidos ( $\mathrm{N}$ e $\mathrm{K}$ ). Além disto, os nutrientes contidos nas folhas, que são móveis no floema, podem ser remobilizados ou redistribuídos para pontos de crescimento e/ ou frutos, antes que as folhas caiam, sendo esta uma estratégia fisiológica das plantas para garantir sua reprodução (Epstein \& Bloom, 2006;).

Uma das razões para a pouca translocação do $\mathrm{Ca}$ pode estar relacionada à sua baixa mobilidade, dificultando sua redistribuição para outras partes da planta antes da queda das folhas (Epstein \& Bloom, 2006). Os teores deste nutriente foram de 38,62 e 38,20 $\mathrm{g} \mathrm{kg}^{-1}$ aos 58 e 107 DAP respectivamente, semelhante aos encontrados por Aquino et al. (2012), outro fato que pode corroborar sua baixa translocação é sua localização na célula que explica, em parte, a limitada redistribuição (Malavolta, 2006). Já o Mg, que é considerado um nutriente de mobilidade alta a intermediária (Marschner, 2002), se comportou de maneira similar ao $\mathrm{Ca}$, apresentando pequena diferença em relação aos teores, tanto na primeira como na segunda época de amostragem (Tabela 3). Segundo Malavolta (2006), é comum encontrar maior ou igual concentração de magnésio tanto em tecidos mais velhos como nos mais jovens, concordando também com os resultados de Veígas et al. (2013).

O resumo da análise de variância apresentado na Tabela 4 denota que os teores foliares dos micronutrientes analisados não tiveram variações significativas entre as épocas amostrais.

Analisando a Tabela 5 é possível concluir que não ocorreu diferença significativa para as diferentes épocas de avaliação foliar dos micronutrientes em análise, de acordo com os valores médios encontrados. Porém, mesmo não sendo constatada significância, pode-se observar grande diferença quando se comparam os valores de Fe nas duas épocas amostrais analisadas.
Tabela 4. Análise de variância dos teores de micronutrientes $\left(\mathrm{mg} \mathrm{kg}^{-1}\right)$, em duas épocas de amostragem, do algodoeiro herbáceo colorido BRS Rubi. Itaporanga-PB, 2009/2010

\begin{tabular}{ccccc}
\hline FV & GL & Fe & Cu & Mn \\
\hline Blocos & 3 & 414,47 & 1,14 & 10,16 \\
Parcelas & 7 & -- & -- & -- \\
Épocas (E) & 1 & $5302,50 \mathrm{~ns}$ & $1,63 \mathrm{~ns}$ & $0,01 \mathrm{~ns}$ \\
Resíduo a & 3 & 616,75 & 0,89 & 12,12 \\
Doses (D) & 5 & $177,35 \mathrm{~ns}$ & $0,49 \mathrm{~ns}$ & $7,40 \mathrm{~ns}$ \\
Int. (E x D) & 5 & $203,55 \mathrm{~ns}$ & $0,44 \mathrm{~ns}$ & $3,49 \mathrm{~ns}$ \\
Resíduo b & 30 & 269,22 & 0,94 & 2,74 \\
Total & 47 & -- & -- & -- \\
Média Geral & -- & 310,2 & 20,95 & 36,5 \\
CV a (\%) & -- & 80,04 & 44,95 & 95,37 \\
CV b (\%) & -- & 52,88 & 46,17 & 45,33 \\
\hline
\end{tabular}

, ns: Significativos a $5 \%$ e $1 \%$ de probabilidade e não significativo, respectivamente

Tabela 5. Médias dos teores de micronutrientes $\left(\mathrm{mg} \mathrm{kg}^{-1}\right)$, em duas épocas de amostragem, do algodoeiro herbáceo colorido BRS Rubi. Itaporanga-PB, 2009/2010

\begin{tabular}{cccc}
\hline Épocas & Fe & Cu & Mn \\
\hline 58 DAP & $415,3 \mathrm{a}$ & $19,1 \mathrm{a}$ & $36,3 \mathrm{a}$ \\
107 DAP & $205,1 \mathrm{a}$ & $22,8 \mathrm{a}$ & $36,7 \mathrm{a}$ \\
\hline
\end{tabular}

Médias seguidas pela mesma letra, nas colunas, não diferem entre si pelo teste de $\mathrm{F}$ a $5 \%$ de probabilidade; $\mathrm{DAP}=$ dias após o plantio

Segundo White (2012); Rosolem et al. (2012), o Fe apresenta mobilidade intermediária no floema, tendendo a diminuir e a aumentar ou permanecer, respectivamente, nos seus teores no decorrer do desenvolvimento reprodutivo vegetal.

Quando o Fe já estiver incorporado nos cloroplastos, ele tem mobilidade bastante limitada dentro das plantas, o que está de acordo com a observação de que os sintomas de deficiência deste micronutriente são restritos às brotações jovens (Carvalho, 2007). Entretanto, Kirkby \& Romheld (2007) relatam que o Fe pode ser translocado através do floema durante a senescência das folhas. Esses autores relatam que o $\mathrm{Fe}$ pode ser redistribuído pelo floema até os ápices das brotações em crescimento sem que haja necessidade de senescência como pré-requisito. Esses dados enfatizam que em princípio, o Fe é móvel no floema, confirmando os valores médios encontrados neste estudo (415,3 e 205,1 $\mathrm{mg} \mathrm{kg}^{-1}$ aos 58 e 107 DAP respectivamente), (Tabela 5).

O Cobre é um nutriente de baixa mobilidade (Epstein \& Bloom, 2006; White, 2012), que pode apresentar de altos a baixos teores nas folhas em crescimento, com mais variações ocasionais e sem tendência definida nos demais estádios fisiológicos.

O manganês apresentou comportamento típico de nutriente de baixa mobilidade, da mesma forma que o $\mathrm{Cu}$, cuja translocação no floema é limitada (Carvalho, 2007), e o teor se incrementou sempre que as plantas se tornaram mais velhas, porém, como os micronutrientes são encontrados em pequenas concentrações nos tecidos vegetais, é recomendável que outras pesquisas sejam desenvolvidas, de preferência em situações de maior controle, objetivando avaliar o efeito da matéria orgânica do solo sobre a absorção desses elementos.

\section{Conclusões}

Dos macronutrientes analisados apenas o $\mathrm{N}$ e $\mathrm{K}$, determinados aos 58 e 107 dias após plantio, apresentaram 
significância e maior redistribuição quando comparados aos demais macronutrientes analisados.

Quanto aos micronutrientes, nenhum se mostrou significativamente diferente quando comparadas as duas épocas de amostragem.

\section{Literatura Citada}

Aquino, L. A. de; Aquino, R. F. B. A.; Silva, T. C.; Santos, D. F. dos; Berger, P. G. Aplicação do fósforo e da irrigação na absorção e exportação de nutrientes pelo algodoeiro. Revista Brasileira de Engenharia Agrícola e Ambiental, v.16, n.4, p.355-361, 2012. <http://dx.doi. org/10.1590/S1415-43662012000400004>.

Brandão, Z. N.; Ferreira, G. B.; Sofiatti, V.; Lima, R. de L. S. de; Medeiros, J. da C. Uso de nitrogênio e fósforo e seus efeitos na nutrição do algodoeiro irrigado. Revista Brasileira de Ciências Agrárias, v.7, n.2, p.213-218, 2012. $<$ http://dx.doi.org/10.5039/agraria.v7i2a1148>.

Camacho, M. A.; Natale, W.; Barbosa, J. C. Faixas de suficiência para a cultura do algodão no centro-oeste do Brasil: I. Macronutrientes, Ciência Rural, v.42, n.8, p.1413-1418, 2012. <http://dx.doi.org/10.1590/S0103$84782012000800013>$.

Carvalho, M. da C. S. Resposta do algodoeiro a fontes, doses e modos de aplicação de zinco no cerrado. Revista Brasileira de Oleaginosas e Fibrosas, v.11, n.3, p.121-129, 2007. <http://www.cnpa.embrapa.br/ojs/index.php/RBOF/ article/view/68/76>. 23 Ago. 2013.

Epstein, E.; Bloom, A. J. Nutrição mineral de plantas. 2.ed. Londrina: Planta, 2006. v.1, 416p.

Garrido, M. da S.; Menezes, R. S. C.; Sampaio, E. V. S. B.; Marques, T. R. R. Crescimento e absorção de nutrientes pelo algodoeiro e pela mamoneira adubados com gliricídia e esterco. Revista Brasileira Engenharia Agrícola e Ambiental, v.13, n.5, p.531-536, 2009. <http://dx.doi. org/10.1590/S1415-43662009000500004>

Kirkby, E. A.; Römheld, V. Micronutrientes na fisiologia de plantas: funções, absorção e mobilidade. Informações agronômicas, n.118, p.1-24, 2007. <http://www.ipni.net/ publication/ia-brasil.nsf/0/8A79657EA91F52F483257AA 10060FACB/\$FILE/Encarte-118.pdf>. 23 Ago. 2013.

Laviola, B. G.; Dias, L. A. dos S. Teor e acúmulo de nutrientes em folhas e frutos de pinhão-manso. Revista Brasileira de Ciência do Solo, v.32, n.5, p.1969-1975, 2008. <http:// dx.doi.org/10.1590/S0100-06832008000500018>.

Laviola, B.G.; Martinez, H.E.P.; Souza, R.B.; Alvarez, V.H. Dinâmica de N e K em folhas, flores e frutos de cafeeiro arábico em três níveis de adubação. Bioscience Journal, v.22, n.3, p.33-47, 2006. <http://www.seer.ufu.br/index. php/biosciencejournal/article/view/6372/4116>. 23 Ago. 2013.

Lima, R. L. S. de; Severino, L. S.; Cazetta, J. O.; Azevedo, C. A. V. de; Sofiatti, V.; Ariel, N. H. C. Redistribuição de nutrientes em folhas de pinhão-manso entre estádios fenológicos. Revista Brasileira de Engenharia Agrícola e Ambiental, v.15, n.11, p.1175-1179, 2011. <http://dx.doi. org/10.1590/S1415-43662011001100010>.
Malavolta, E. Manual de nutrição mineral de plantas. São Paulo: Ceres, 2006. 638p.

Malavolta, E.; Nogueira, N. G. L.; Heinrichs, R.; Higashi, E. N.; Rodriguez, V.; Guerra, E.; Oliveira, S. C.; Cabral, C. P. Evaluation of nutritional status of the cotton plant with respect to nitrogen. Communications in Soil Science and Plant Analysis, v.35, n.7, p.1007-1019, 2004. < http:// dx.doi.org/10.1081/CSS-120030577>.

Marenco, R. A.; Lopes, N. F. Fisiologia vegetal: fotossíntese, respiração, relações hídricas e nutrição mineral. Viçosa Universidade Federal de Viçosa, 2005. 451p.

Marschner, H. Mineral nutrition of higher plants. London: Academic Press, 2002. 889p.

Morais, D. L. de. Impacto da nutrição mineral no crescimento do pinhão manso (Jatropha curcas L.). Patos: Universidade Federal de Campina Grande, Centro de Saúde e Tecnologia Rural, 2010. 54p. Dissertação Mestrado. <http://www.cstr. ufcg.edu.br/ppgcf/Dissertacoes/dissert_deborah.pdf>. 23 Ago. 2013.

Motomiya, A. V. de A.; Molin, J. P.; Motomiya, W. R.; Biscaro, G. A. Diagnose nutricional com o uso de sensor óptico ativo em algodoeiro. Revista Brasileira de Engenharia Agrícola e Ambiental, v.16, n.11, p.1159-1165, 2012. <http://dx.doi. org/10.1590/S1415-43662012001100003>.

Politi, L. S.; Flores, R. A.; Silva, J. A. S. da; Wadt, P. G. S.; Pinto, P. A. da C.; Prado R. de M. Estado nutricional de mangueiras determinado pelos métodos DRIS e CND. Revista Brasileira de Engenharia Agrícola e Ambiental, v.17, n.1, p.11-18, 2013. <http://dx.doi.org/10.1590/ S1415-43662013000100002>.

Ramos, A. M.; Santos, L. A. R. dos; Fortes, L. T. G. Normas climatológicas do Brasil: 1961-1990. Brasília: INMET, 2009. 465p.

Rosolem, C. A.; Deus, A. C. F.; Martins, P. O.; Léles, E. P. Acúmulo e distribuição de boro em cultivares de algodão. Revista Brasileira de Ciência do Solo, v.36, n.4 p.1231-1238, 2012. <http://dx.doi.org/10.1590/S0100$06832012000400017>$.

Rosolem, C.A., Silva, R.H.; Esteves, J.A.F. Suprimento de potássio a raízes de algodoeiro em razão da adubação potássica e calagem. Pesquisa Agropecuária Brasileira, v.38, n.5, p.635-641, 2003. <http://dx.doi.org/10.1590/ S0100-204X2003000500012>.

Serra, A. P.; Marchetti, M. E.; Vitorino, A. C. T.; Novelino, J. O.; Camacho, M. A. Determinação de faixas normais de nutrientes no algodoeiro pelos métodos ChM, CND e DRIS. Revista Brasileira de Ciência do Solo, v.34, n.1, p.105-113, 2010a. <http://dx.doi.org/10.1590/S0100$06832010000100011>$.

Serra, A. P.; Marchetti, M. E.; Vitorino, A. C. T.; Novelino, J. O.; Camacho, M. A. Desenvolvimento de normas DRIS e CND e avaliação do estado nutricional da cultura do algodoeiro. Revista Brasileira de Ciência do Solo, v.34, n.1, p.97-104, 2010b. <http://dx.doi.org/10.1590/S0100$06832010000100010>$. 
Severino, L.S.; Costa, F.X.; Beltrão, N.E. de M.; Lucena, A.M.A. de; Guimaraes, M.M.B. Mineralização da torta de mamona, esterco bovino e bagaço de cana estimada pela respiração microbiana. Revista de Biologia e Ciências da Terra, v.5, n.1, 2004. <http://eduep.uepb.edu.br/rbct/ sumarios/pdf/esterco.pdf>. 23 Ago. 2013.

Silva, F. C. da. Manual de análises químicas de solos, plantas e fertilizantes. 2. ed. Brasília: Embrapa Informação Tecnológica, 2009. $627 \mathrm{p}$.

Silva, F. de A. S.; Azevedo, C. A. V. de. Versão do programa computacional assistat para o sistema operacional Windows. Revista Brasileira de Produtos Agroindustriais, v.4, n.1, p.71-78, 2002. <http://www.deag.ufcg.edu.br/ rbpa/rev41/Art410.pdf>. 23 Ago. 2013.

Silva, M. A. C. da. Métodos de avaliação do estado nutricional para o algodoeiro no Centro-Oeste do Brasil. Jaboticabal: Universidade Estadual Paulista, Faculdade de Ciências Agrárias e Veterinárias, 2006. 75p. Tese Doutorado. $<$ http://www.fcav.unesp.br/download/pgtrabs/pv/d/1636. pdf $>.23$ Ago. 2013.

Silva, N. M. Calagem e adubação do algodoeiro, In: seminário estadual com a cultura do algodão, 3, 1996. Cuiabá: Empaer, 1996. 176p.
Sousa, A. E. C.; Lacerda, C. F. de; Gheyi, H. R.; Soares, F. A. L.; Uyeda, C. A. Teores de nutrientes foliares e respostas fisiológicas em pinhão manso submetido a estresse salino e adubação fosfatada. Revista Caatinga, v.25, n.2, p.144-152, 2012. <http://periodicos.ufersa. edu.br/revistas/index.php/sistema/article/view/2291/ pdf>. 23 Ago. 2013.

Souto, P.C.; Souto, J.S.; Santos, R.V.; Araújo, G.T.; Souto, L.S. Decomposição de estercos dispostos em diferentes profundidades em área degradada no semiárido da Paraíba. Revista Brasileira de Ciência do Solo, v.29, n.1 p.125-130， 2005. <http://dx.doi.org/10.1590/S010006832005000100014>.

Veígas, I. de J. M.; Sousa, G. O. de; Silva, A. F. da; Carvalho, J. G. de; Lima, M. M. Composição mineral e sintomas visuais de deficiências de nutrientes em plantas de pimenta-longa (Piper hispidinervum C. DC.). Acta Amazônica, v.43, n.1, p.43-50, mar. 2013. <http://dx.doi. org/10.1590/S0044-59672013000100006>.

White, P. J. Long-distance transport in the xylem and phloem. In: Petra, M. (Ed.). Marschener's mineral nutrition of higher plants. New York: Academic Press, 2012. Cap.3, p.49-70. 